\title{
Multifractality in the general cosmological solution of Einstein's equations
}

\author{
John D. Barrow \\ DAMTP, Centre for Mathematical Sciences, \\ University of Cambridge, \\ Wilberforce Road, Cambridge CB3 0WA \\ United Kingdom
}

July 22,2020

\begin{abstract}
We demonstrate the scale invariance of the vacuum Bianchi type IX equations and use this to argue for the possibility of multifractal turbulence as a realisation of the suggestion by Belinski that there will be a fragmentation of local regions of inhomogeneous Mixmaster chaos on approach to an initial inhomogeneous cosmological singularity. Differences between the gravitational and hydrodynamical situations are outlined. Various potential obstacles to this picture of gravitational turbulence are discussed together with links to preinflation.
\end{abstract}

\section{Introduction}

We have recently explored the potential effects of synchronisation of Mixmaster oscillations between different regions of an inhomogeneous Mixmaster universe on approach to the initial cosmological singularity [1]. Here, we consider another generalisation of the standard picture of Bianchi type IX evolution towards the singularity in the inhomogeneous generalisation. Belinski 2 has suggested that there could be further added structure to the general inhomogeneous Mixmaster oscillations in the form of the transport of energy from large to small scales leading (like in any complex nonlinear system with infinite number of degrees of freedom) to the endless generation of excitations of smaller and smaller spatial scales. This process can be called the fragmentation of the Mixmaster oscillatory regime and its rise is supported by the mathematical fact of the monotonic increase of spatial gradients of the metric on approach to the singularity that was observed by Kirillov and Kochnev [7, 8, and by Montani 9, 10. A further discussion of this fragmentation idea appears in the book [4, see also ref. [6].

This phenomenon is reminiscent of three-dimensional fluid turbulence but with no minimum dissipative scale and so the energy transport continues down 
to zero scale. The approach to the singularity at $t=0$ produces an infinite number of subdivisions of each local inhomogeneous Mixmaster dynamical region and fragmentation continues ad infinitum. There will only be a cut-off to this process and to the downward energy transfer if there is a minimum length scale where dissipation of the gravitational wave energy can occur; by particle production of gravitons, or other degrees of freedom; or if there is a bounce [11, 12] at a minimum non-zero radius; or if the oscillations cease because of asymptotic dominance of the local dynamics by a massless scalar field or the kinetic energy of a scalar field with a non-zero potential.

Another ingredient that is potentially relevant, and maybe even related, are spikes and their oscillations, [13, 14. In the next section we provide some more rigorous basis for our

\section{Type IX scaling and multifractal behaviour}

The spatially homogeneous diagonal Bianchi IX metric is,

$$
d s^{2}=d t^{2}-\gamma_{a b}(t) e_{\mu}^{a} e_{\nu}^{b} d x^{\mu} d x^{\nu}
$$

where

$$
\gamma_{a b}(t)=\operatorname{diag}\left[a^{2}(t), b^{2}(t), c^{2}(t)\right]
$$

and

$$
e_{\mu}^{a}=\left(\begin{array}{ccc}
\cos z & \sin z \sin x & 0 \\
-\sin z & \cos z \sin x & 0 \\
0 & \cos x & 1
\end{array}\right)
$$

The general relativistic field equations in vacuum Bianchi type IX with scale factors $a(t), b(t), c(t)$ in comoving proper time $t,[3,4$, 5, are:

$$
\begin{gathered}
(\dot{a} b c)^{\cdot}=\frac{1}{2 a b c}\left[\left(b^{2}-c^{2}\right)^{2}-a^{4}\right] \\
(a \dot{b} c)^{\cdot}=\frac{1}{2 a b c}\left[\left(a^{2}-c^{2}\right)^{2}-b^{4}\right] \\
(a b \dot{c})^{\cdot}=\frac{1}{2 a b c}\left[\left(a^{2}-b^{2}\right)^{2}-c^{4}\right] \\
\left(\frac{\dot{a}}{a}+\frac{\dot{b}}{b}+\frac{\dot{c}}{c}\right)^{2}=\left(\frac{\dot{a}}{a}\right)^{2}+\left(\frac{\dot{b}}{b}\right)^{2}+\left(\frac{\dot{c}}{c}\right)^{2}+ \\
+\frac{1}{2 a^{2} b^{2} c^{2}}\left(a^{4}+b^{4}+c^{4}-2 a^{2} b^{2}-2 a^{2} c^{2}-2 b^{2} c^{2}\right) .
\end{gathered}
$$

Eqn. (7) is also expressed more simply before integration as, 


$$
\frac{1}{a} \frac{d^{2} a}{d t^{2}}+\frac{1}{b} \frac{d^{2} b}{d t^{2}}+\frac{1}{c} \frac{d^{2} c}{d t^{2}}=0 .
$$

Now consider the scaling behaviour of these equations by a power, $h$, of a constant positive scaling factor $A$. We set

$$
\begin{aligned}
(a, b, c) & \rightarrow A^{h}\left(a_{*}, b_{*}, c_{*}\right), \\
t & \rightarrow A^{h} t_{*} .
\end{aligned}
$$

Then, eqn. (44) transforms in the $*$ variables to (where ${ }^{\prime}$ denotes $d / d t_{*}$ ),

$$
A^{h}\left(a_{*}^{\prime} b_{*} c_{*}\right)^{\prime}=\frac{A^{h}}{2 a_{*} b_{*} c_{*}}\left[\left(b_{*}^{2}-c_{*}^{2}\right)^{2}-a_{*}^{4}\right],
$$

for arbitrary constants, $A$ and $h$. The same invariance holds for eqns.(15)-(6) by cyclically permuting letters $a, b$, and $c$. The first integral,eq.(17), transforms as,

$$
\begin{gathered}
\frac{1}{A^{2 h}}\left(\frac{a_{*}^{\prime}}{a_{*}}+\frac{b_{*}^{\prime}}{b_{*}}+\frac{c_{*}^{\prime}}{c_{*}}\right)^{2}=\frac{1}{A^{2 h}}\left[\left(\frac{a_{*}^{\prime}}{a_{*}}\right)^{2}+\left(\frac{b_{*}^{\prime}}{b_{*}}\right)^{2}+\left(\frac{c_{*}^{\prime}}{c_{*}}\right)^{2}\right] \\
+\frac{1}{2 a_{*}^{2} b_{*}^{2} c_{*}^{2} A^{2 h}}\left(a_{*}^{4}+b_{*}^{4}+c_{*}^{4}-2 a_{*}^{2} b_{*}^{2}-2 a_{*}^{2} c_{*}^{2}-2 b_{*}^{2} c_{*}^{2}\right),
\end{gathered}
$$

and again we see it has the same invariance, as expected, because eq. (77) is a first integral of eqns. (4)-(6), and the equation is scale invariant under all $A$ and $h$ scaling transformations. The same scaling is seen more obviously from eqn. (81). The 'toy' type IX model created by Fleig and Belinski [15] does not have this scaling property. This is not surprising as the equations were created by setting $c(t)=0$ and so the scale invariance in eq. (9) is obviously broken.

The scale invariance of these equations has important consequences. Parisi and Frisch 16, 17, 18 famously considered a similar problem in the context of scale invariance of the Euler and Navier-Stokes equations and its relation to the presence of turbulence with multifractal structure. The scale invariance property of the vacuum type IX equations we have shown means that in the inhomogeneous generalisation of the Mixmaster model there can exist many different values of $h$, each occurring say with some probability $P_{r}(h)$ on any fixed scale $r$. So, a turbulent situation appears as a superposition of many different scale-invariant flows. In order to maintain scale invariance when averaging correlation functions one need $P_{r}(h) \simeq r^{f(h)}$, for some unknown function $f(h)$ so that the scale invariance holds for the distribution $P_{r}(h)$ also. Parisi and Frisch took $f(h)$ to be $3-D(h)$, with $D(h)$ taken to be the fractal (Hausdorff) dimension of the scale invariant solution with scaling exponent $h$. Hence, this scenario is referred to as multifractal turbulence and we see that it arises in the inhomogeneous extension of the vacuum Bianchi type IX equations. In the hydrodynamic problem, a further constraint is introduced by using the Kolmogorov constraint that there is scale-invariant energy flow per unit time from 
large to small scales. This requires constant energy flow rate, $v^{2} / t \simeq v^{3} / r$, through scale $r$, so $v \propto r^{1 / 3}$ independent of the scaling parameters: the famous Kolmogorov spectrum.

We know that a turbulent inertial region requires a lower length limit cut-off where dissipation of the energy injected on large scales can occur. Of course, in the hydrodynamical case this scale breaks the scale invariance but the situation may be simpler in the cosmological case as it is not obvious if a lower length scale exists as a cut-off to the turbulent fragmentation. There are three simple possibilities:

a. The lower length cut-off is the Planck scale, $l_{p l} \simeq 10^{-33} \mathrm{~cm}$. However, if this is the case there will be a very small number of oscillations (of order 10) between the present and the Planck time (a mean expansion scale factor change of order $10^{60}$ ) as they occur in $\ln (\ln t)$ time - far too few to set up a well-developed inertial regime for some type of gravitational turbulence.

b. The fragmentation degenerates into the formation of some organized objects, for example, as gravitational solitonic structures in which there is a balance between gravity and dispersion, which act a limiting case [19, 20. They may also be related to the synchronisation process outlined in ref. [1. This scenario also appears in other aspects of hydrodynamic turbulence studies. The Kolmogorov-Hinze theory of the fragmentation of droplets in a chaotic turbulent flow is relevant 22, 23,. Using Kolmogorov's classic velocity spectrum, they derived a criterion for the maximum size of droplet that will not undergo fragmentation when the turbulent flow intensity exceeds the surface tension 24]. Many theoretical and experimental studies have been made of the details of this process and the generalisations and limitations of the Kolmogorov-Hinze picture [25]. It is suggestive of an analogy in the cosmological picture where Weyl curvature oscillations overcome self-gravity to perpetuate anisotropic stretching and fragmentation of regions.

b. There is no lower limit and the chaotic oscillations and turbulent behaviour continue all the way to $t=0$. This results in an infinite number of spacetime oscillations of the scale factors $a, b, c$ in $t$ time (or equivalently $a_{*}, b_{*}, c_{*}$ in $t_{*}$ time) but with the conceptual problem of what is the meaning of the model at times earlier than the Planck time when less than one photon is present inside the horizon and so statistical mechanics is meaningless (and perhaps the concept of space and time oscillations as well (but see ref. 21]) in a non-quantum gravitational form.

We should also mention the potential links to the study of the pre-inflationary structure of the very early universe, see for example ref. 26. Preinflation can imprint particular fluctuation scales when density perturbations cross the horizon and provide a way to access information about the preinflationary (possibly chaotic) structure of the very early universe. Our study has focussed on the multifractal evolution on approach to an initial singularity $(t \rightarrow 0)$ and so any remnant of preinflation has to look at the evolution in the opposite time-sense where the fragmentation process will run in reverse. Again, a we have stressed above, any interval of Mixmaster evolution not including $t=0$ creates a finite number of oscillations and fragmentations and so as $t$ grows there may 
be a residual effect of early fragmentation but it will not have been in the fully developed gravitational turbulence range. This may repay further investigation.

In this paper we have tried to provide some simple underpinning for the idea that there is random fragmentation of Mixmaster dynamics in different regions on approach to the singularity in an inhomogeneous version of the Bianchi type IX universe when the inhomogeneities are still not too large.

Acknowledgements. I would like to thank V.A. Belinski for encouragement, discussions and detailed comments. Support by the Science and Technology Facilities Council (STFC) of the UK is acknowledged.

\section{References}

[1] J.D. Barrow, arXiv 2006.01562

[2] V.A. Belinski, Sov. Phys. JETP Lett. 56, 421 (1992)

[3] L. Landau and E.M. Lifshitz, The Classical Theory of Fields, Pergamon, Oxford, 4th rev edn., (1974)

[4] V.A. Belinski and M. Henneaux, The Cosmological Singularity, Cambridge Univ. Press, Cambridge, pp. 53-56 (2018)

[5] C. W. Misner, Phys. Rev. Lett. 22, 1071 (1969)

[6] J.D. Barrow, Phys. Reports 85, 1 (1982)

[7] A. A. Kirillov and A. A. Kochnev, Sov. Phys. JETP Lett. 46, 436 (1987)

[8] A. A. Kirillov, Sov. Phys. JETP 76, 355 (1993)

[9] G. Montani, M.V. Battisti, R. Benini, and G. Imponente, Int. J. Mod. Phys. A 23, 2353, (2008) arXiv:0712.3008

[10] G. Montani, Classical and Quantum Gravity 12, 2505 (1995)

[11] J.D. Barrow and C. Ganguly Phys. Rev. D 95, 083515 (2017)

[12] C. Ganguly and J.D. Barrow, Phys. Rev. D 96, 123534 (2017)

[13] W.C. Lim, Classical and Quantum Gravity 25, 045014 (2008)

[14] J.M. Heinzle, C. Uggla, and W.C. Lim, Phys. Rev. D 86 ,104049 (2012)

[15] P. Fleig and V.A. Belinski, arxiv 1811.05208

[16] Frisch, U., Parisi, G. On the singularity structure of fully developed turbulence. In Turbulence and Predictability in Geophysical Fluid Dynamics and Climate Dynamics, ed. M. Ghil, R. Benzi, G. Parisi, pp. 84-88, Amsterdam: North-Holland (1985) 
[17] Benzi, R., Paladin, G., Parisi, G., Vulpiani, A. On the multifractal nature of fully developed turbulence and chaotic systems. J. Phys. A 17, 3521 (1984)

[18] Frisch, U., Fully Developed Turbulence and Intermittency, In Turbulence and Predictability in Geophysical Fluid Dynamics and Climate Dynamics, ed. M. Ghil, R. Benzi, G. Parisi, pp. 84-88, Amsterdam: North-Holland (1985), with an Appendix by G. Parisi and U. Frisch, On the singularity structure of fully developed turbulence, pp.84-87

[19] V.A. Belinski and V.E. Zakharov, Sov. Phys. JETP 48, 985 (1978)

[20] V.A. Belinski and E. Verdaguer, Gravitational Solitons, Cambridge Univ. Press, Cambridge (2001)

[21] C.W. Misner, Phys. Rev. 186, 1328 (1969)

[22] A.N. Kolmogorov, Dokl. Akad. Nauk. SSSR, 66, 825 (1949)

[23] J.O. Hinze, A. I. Ch.E. Journal 1, 289 (1955)

[24] P. Perlekar, L. Biferale, M. Sbragaglia, S. Srivastava and F. Toschi, https://arxiv.org/abs/1112.6041

[25] C. D. Eastwood, L. Armi and J. C. Lasheras, J. Fluid Mech. 502, 309 (2004)

[26] G. J. Mathews, I.-S. Suh, N. Q. Lan and T. Kajino, Phys. Rev. D 92,123514 (2015) 